\title{
NOTES ON A PAPER BY J. B. MILLER
}

\author{
J. L. LAVOIE ${ }^{1}$
}

(Received 2 June 1986)

\begin{abstract}
Two sums given by J. B. Miller are evaluated in terms of classical hypergeometric results.
\end{abstract}

\section{Introduction}

In a recent paper [2] on the foliage density equation, J. B. Miller reproduces a proof due to G. A. Watterson, of the relation

$$
\sum_{l=0}^{j}\left(\begin{array}{c}
2 k \\
2 l
\end{array}\right)\left(\begin{array}{c}
k-l \\
k-j
\end{array}\right)=\frac{\dot{k}}{j}\left(\begin{array}{c}
k+j-1 \\
k-j
\end{array}\right) 2^{2 j-1}, \quad 0 \leqslant j \leqslant k .
$$

He also considers the sum

$$
S_{r}(j)={ }_{3} F_{2}\left(\begin{array}{cc}
-r, r+2 j, & j \\
j-\frac{1}{2}, & 2 j+2
\end{array} \mid 1\right)
$$

and says that by using a Burroughs B6700 computer B. J. Milne has obtained the following formulae, valid at least for $r=0,1, \ldots, 12$ and all $j \geqslant 1$. There are separate forms for $r=2 s+1$ and $r=2 s$, with integral $s \geqslant 1$;

$$
\begin{aligned}
& S_{2 s+1}(j)= \\
& -\frac{1^{2} \cdot 3^{2} \cdot 5^{2} \cdots(2 s-1)^{2}(2 s+1)}{2^{2 s+1}\left(j^{2}-\frac{1}{4}\right)(j+1)\left(j+\frac{3}{2}\right)^{2}\left(j+\frac{5}{2}\right)^{2} \cdots\left(j+\frac{2 s-1}{2}\right)^{2}\left(j+\frac{2 s+1}{2}\right)},
\end{aligned}
$$

\footnotetext{
${ }^{1}$ Département de mathématiques, statistique et actuariat, Université Laval, Québec, Canada, GIK 7 P4.

(1) Copyright Australian Mathematical Society 1987, Serial-fee code 0334-2700/87
} 


$$
S_{2 s}(j)=S_{2 s+1} \cdot \frac{2 j^{2}+(4 s+1) j+4 s^{2}-1}{(2 s-1)(2 s+1)}, \quad s=1,2,3, \ldots
$$

\section{Some hypergeometric results}

a) Note that (1) is a special case of Gauss's classical result

$$
{ }_{2} F_{1}\left(\begin{array}{ll}
a, & b \\
c & 1
\end{array}\right)=\frac{\Gamma(c) \Gamma(c-a-b)}{\Gamma(c-a) \Gamma(c-b)}, \quad R(c-a-b)>0 .
$$

More explicitly, for $0 \leqslant j \leqslant k$, we have

$$
\sum_{l=0}^{j}\left(\begin{array}{c}
2 k \\
2 l
\end{array}\right)\left(\begin{array}{l}
k-l \\
k-j
\end{array}\right)=\left(\begin{array}{l}
k \\
j
\end{array}\right)_{2} F_{1}\left(\begin{array}{cc}
-j, & \frac{1}{2}-k \\
\frac{1}{2}
\end{array} \mid 1\right)=\frac{(k+j-1) ! k}{j !(k-j) !\left(\frac{1}{2}\right)_{j}}
$$

where

$$
(\lambda)_{n}=\frac{\Gamma(\lambda+n)}{\Gamma(\lambda)}=\left\{\begin{array}{l}
1, \quad \text { if } n=0, \\
\lambda(\lambda+1) \cdots(\lambda+n-1), \quad \forall n \in\{1,2, \ldots\} .
\end{array}\right.
$$

b) Instead of (2), consider the more general sum

$$
S(a, b, c)={ }_{3} F_{2}\left(\begin{array}{lc}
a, & b, \\
\frac{1}{2}(a+b-1), & 2 c+2
\end{array} \mid 1\right) .
$$

Rainville [3], pp. 81-85, has given many relations involving contiguous functions, one of the simplest of which is

$$
(\alpha-\beta+1) F=\alpha F(\alpha+1)-(\beta-1) F(\beta-1) .
$$

Letting

$$
F={ }_{3} F_{2}\left(\begin{array}{lc|}
a, \quad b, & c \\
\frac{1}{2}(a+b+1), & 2 c+2
\end{array} \mid 1\right)
$$

then for $\alpha=c$ and $\beta=\frac{1}{2} a+\frac{1}{2} b+\frac{1}{2}$, we obtain

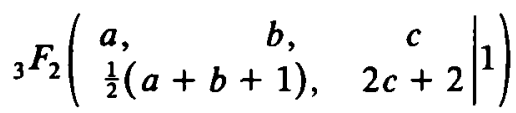

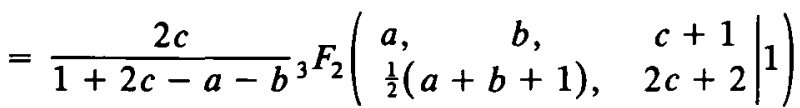

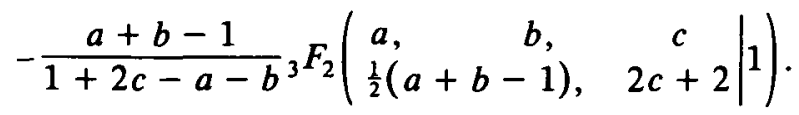


Also, when $\alpha=c$ and $\beta=2 c+2$, we find that

$$
\begin{aligned}
& { }_{3} F_{2}\left(\begin{array}{cc|c}
a, & b, & c \\
\frac{1}{2}(a+b+1), & 2 c+2
\end{array}\right)
\end{aligned}
$$

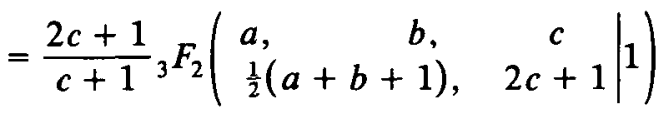

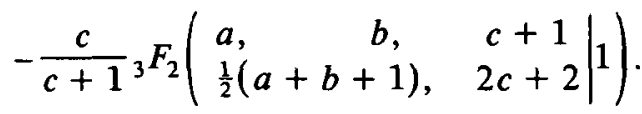

Equating the right-hand sides of these last two relations yields

$$
\begin{aligned}
& \frac{a+b-1}{1+2 c-a-b} S(a, b, c)
\end{aligned}
$$

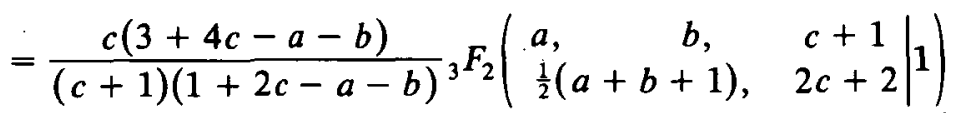

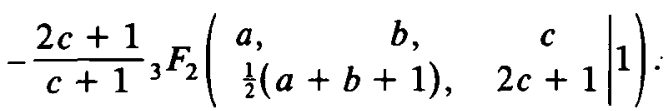

Finally, the left hand side can be easily transformed into the right hand side in the following relation:

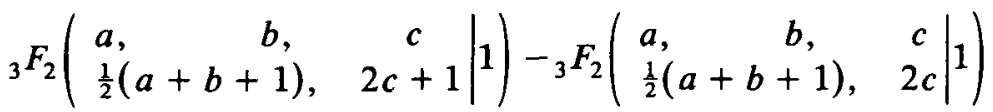

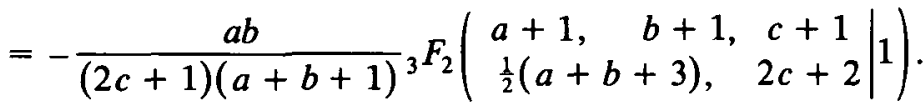

Using this in the right side of (6) yields

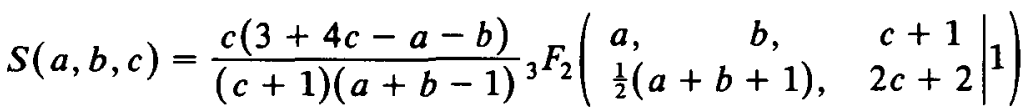

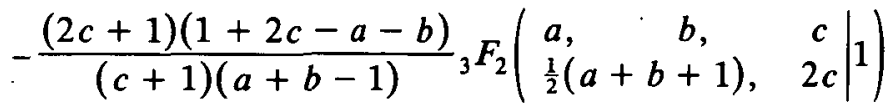

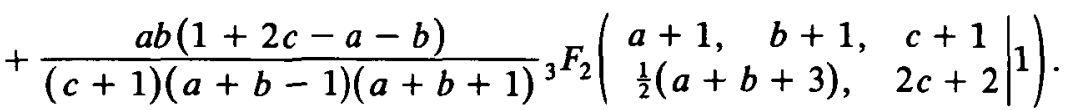

The three ${ }_{3} F_{2}$, on the right of the last relation, can be evaluated by Watson's classical summation formula [1], p. 16, 3.3.1:

$$
\begin{aligned}
{ }_{3} F_{2}\left(\begin{array}{cc|c}
a, & b, & c \\
\frac{1}{2}(a+b+1), & 2 c & 1
\end{array}\right) \\
=\frac{\Gamma\left(\frac{1}{2}\right) \Gamma\left(\frac{1}{2} a+\frac{1}{2} b+\frac{1}{2}\right) \Gamma\left(c+\frac{1}{2}\right) \Gamma\left(c-\frac{1}{2} a-\frac{1}{2} b+\frac{1}{2}\right)}{\Gamma\left(\frac{1}{2} a+\frac{1}{2}\right) \Gamma\left(\frac{1}{2} b+\frac{1}{2}\right) \Gamma\left(c-\frac{1}{2} a+\frac{1}{2}\right) \Gamma\left(c-\frac{1}{2} b+\frac{1}{2}\right)} .
\end{aligned}
$$


Thus

$$
\begin{array}{r}
S(a, b, c)=\frac{\Gamma\left(\frac{3}{2}\right) \Gamma\left(\frac{1}{2} a+\frac{1}{2} b-\frac{1}{2}\right) \Gamma\left(c+\frac{3}{2}\right) \Gamma\left(c-\frac{1}{2} a-\frac{1}{2} b+\frac{3}{2}\right)}{(c+1) \Gamma\left(\frac{1}{2} a+\frac{1}{2}\right) \Gamma\left(\frac{1}{2} b+\frac{1}{2}\right)} \\
\times\left\{\frac{a b \Gamma\left(\frac{1}{2} a+\frac{1}{2}\right) \Gamma\left(\frac{1}{2} b+\frac{1}{2}\right)}{\Gamma\left(\frac{1}{2} a+1\right) \Gamma\left(\frac{1}{2} b+1\right) \Gamma\left(c-\frac{1}{2} a+1\right) \Gamma\left(c-\frac{1}{2} b+1\right)}\right. \\
\left.-\frac{(1-a)(1-b)+(1-a-b) c}{\Gamma\left(c-\frac{1}{2} a+\frac{3}{2}\right) \Gamma\left(c-\frac{1}{2} b+\frac{3}{2}\right)}\right\}, \\
R(2 c-a-b)>-3 .
\end{array}
$$

In particular, from (2), (5) and (7):

$$
\begin{aligned}
S_{2 s+1}(j) & =S(-1-2 s, 2 s+2 j+1, j) \\
& =-\frac{\left(s+\frac{1}{2}\right)\left(s+j+\frac{1}{2}\right)\left(\frac{1}{2}\right)_{s}\left(\frac{1}{2}\right)_{s}}{(j+1)\left(j^{2}-\frac{1}{4}\right)\left(j+\frac{3}{2}\right)_{s}\left(j+\frac{3}{2}\right)_{s}},
\end{aligned}
$$

which is (3), and (4) is similarly obtained.

\section{Some other sums}

The expressions

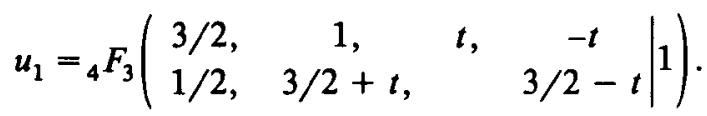

and

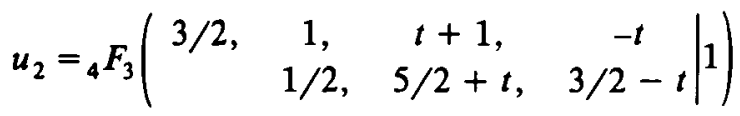

are found in (45) and (46) of Miller's paper and they can be evaluated. These are terminating Saalschützian ${ }_{4} F_{3}$ and they can be transformed by the following formula, [1], p. 56, 7.2.1:

$$
\begin{aligned}
& { }_{4} F_{3}\left(\begin{array}{c}
x, y, z,-n \\
u, v, w
\end{array}\right) \\
& \quad=\frac{(v-z)_{n}(w-z)_{n}}{(v)_{n}(w)_{n}}{ }_{4} F_{3}\left(\begin{array}{c}
u-x, u-y, \quad z,-n \\
1-v+z-n, 1-w+z-n, u
\end{array} \mid 1\right) .
\end{aligned}
$$

With

$$
x=3 / 2, \quad y=1, \quad z=t, \quad n=t, \quad u=1 / 2, \quad v=3 / 2+t, \quad w=3 / 2-t,
$$


the ${ }_{4} F_{3}$ on the right will contain only two non zero terms and we find that

$$
u_{1}=\frac{\left(1-4 t^{2}\right)\left(1-8 t^{2}\right)}{1-16 t^{2}} \text {. }
$$

We find also, in exactly the same way, that

$$
u_{2}=-\frac{(2 t-1)(2 t+3)}{(4 t+1)(4 t+3)}\left(8 t^{2}+8 t+1\right)
$$

\section{References}

[1] W. N. Bailey, Generalized hypergeometric series (Stechert-Hafner Service Agency, New York and London, 1964).

[2] J. B. Miller, "The foliage density equation revisited," J. Austral. Math. Soc. Ser. B 27 (1986) 387-401.

[3] E. D. Rainville, Special functions (The Macmillan Company, New York, 1960). 